\title{
WAKE VORTEX EFFECTS ON PARALLEL RUNWAY OPERATIONS
}

\author{
J. N. Hallock ${ }^{*}$ and S. P. Osgood ${ }^{\dagger}$ \\ John A. Volpe National Transportation Systems Center \\ Cambridge, MA 02142 \\ J. Konopka \\ DFS Deutsche Flugsicherung GmbH \\ D-63225 Langen, Germany
}

\begin{abstract}
Aircraft wake vortex behavior in ground effect between two parallel runways at Frankfurt/Main International Airport was studied. The distance and time of vortex demise were examined as a function of crosswind, aircraft type, and a measure of atmospheric turbulence. Vortex decay in ground effect is little influenced by ambient turbulence and is seen to be a stochastic process.
\end{abstract}

\section{INTRODUCTION}

Air traffic control employs aircraft-to-aircraft separation standards to prevent both collisions and potentially hazardous wake turbulence encounters. Separation standards depend on the phase of flight (takeoff, cruise, landing) and the geometry of the airspace involved (holding patterns, single runway operations, parallel runway operations, etc.). The separations depend on radar coverage and the aircraft categories (e.g., Heavy, B-757, Large, and Small in the United States and Heavy, Medium, and Light in Germany). The categories are based on the maximum certificated gross takeoff weight.

Extensive wake vortex data have been collected at many airports (Kennedy ${ }^{1}$, Stapleton ${ }^{2}$, O'Hare ${ }^{3}$, Toronto $^{4}$, Memphis $^{5}$, Los Angeles $^{6}$, Heathrow ${ }^{7}$, Schiphol $^{8}$, Dallas/Ft. Worth ${ }^{9}$, and Toulouse ${ }^{10}$ ). In these efforts, the intent was to study vortex behavior near or over a single runway during landing or takeoff. Current regulations, however, require wake turbulence separation for aircraft operating on parallel runways if the separation of the runways is less than 760 meters. Data collection at Frankfurt $^{11}$ and San Francisco ${ }^{12}$ addressed the parallel runway situation.

The measurements at San Francisco (SFO) are continuing and the vortex behavior near runway threshold and along the runway is being examined. The runways at SFO are separated by 137 meters.
The measurements at Frankfurt/Main (FRA) are also continuing and the vortex behavior at a nominal distance of 900 meters from the threshold of runways $25 \mathrm{~L}$ and 25R (907 meters and 874 meters, respectively) is under study. The runways at FRA are separated by 518 meters. The measurements at FRA can be grouped into two campaigns. The first was conducted in the 1980s and was directed at the development of a Wake Vortex Warning System ${ }^{13,14}$ (WVWS) to increase the capacity of the parallel runways through a decrease in the required wake turbulence separations for staggered approaches under certain meteorological conditions. The second campaign, and the subject of this paper, came about when the sensors of the WVWS were used to collect wind data to develop a statistical persistence model to predict the crosswind within a 20 -minute period ${ }^{15}$. While the anemometer array of the WVWS aimed at an accurate measurement of local wind and turbulence in the vicinity of the runway thresholds, subsequent analyses showed that the anemometer array served as a vortex detector, too. Wind and wake vortex data from 1997 to the present have been recorded.

The FRA data consists of five years of aircraft landings on runways $25 \mathrm{~L}$ and $25 \mathrm{R}$. Most interesting from a wake vortex perspective is the fact that almost $30 \%$ of the aircraft operating at Frankfurt fall in the Heavy category. Automated analysis routines need to be developed to handle this amount of data. Such routines are in development. To "calibrate" the routines, the data collected in April 1998 was

\footnotetext{
${ }^{*}$ Manager, Aviation Safety Division, Senior Member, AIAA

${ }^{\dagger}$ Computer Specialist

†Project Manager, Wake Vortex Warning System
} 
processed using both semi-automated routines and manual processing. This paper reports on the in-depth analysis of the April 1998 data and first results about vortex lifetimes obtained using more data with automated routines.

\section{DATA COLLECTION}

\subsection{Test Site}

The data collection site is located on the eastern end of the parallel runways at FRA. The runways are oriented at $250^{\circ}$ magnetic and labeled $25 \mathrm{~L}$ and $25 \mathrm{R}$. The runways are separated by 518 meters. The threshold of runway $25 \mathrm{~L}$ is displaced 228 meters to the west of the threshold of 25R. Both runways have an Instrument Landing System with a glide slope angle of $3^{\circ}$.

\subsection{Anemometer Array}

Diagnosing and predicting wake vortex motion in a Wake Vortex Warning or Advisory System - to a considerable extent - relies on the measured and predicted winds. For this purpose, an array of anemometers was installed as one component of the WVWS. Because of the taxiways and the Autobahn A5, placement of the sensors was restricted. The array was installed at an angle so that each end of the array was about the same distance from the runway threshold. To the south of the test site the area was relatively flat. To the north is the commercial terminal and freight facilities.

Ten three-axis ultrasonic METEK USA-1 anemometers are mounted on 15-meter masts. The masts are separated by 50 meters. The wind data were recorded with a sampling rate of $25 \mathrm{~Hz}$, but the analyses herein used $0.5 \mathrm{~Hz}$ resolution only. Reference 13 describes the data recording.

At the location of the anemometer array the aircraft passed overhead at a nominal height of 65 meters. Thus, the Heavy aircraft were about 1 wingspan above the ground and the Large/Medium aircraft were about 2 wingspans above the ground.

\section{DATA REDUCTION}

As noted above, the data collected in April 1998 was selected for detailed reduction and analysis. The aircraft types of the aircraft landing on runways $25 \mathrm{~L}$ and 25R along with the landing times were obtained from the airport runway logs. Only data where the aircraft type was known was reduced.
Daily data files ${ }^{16}$ were established containing 2second averages of the ultrasonic anemometer measurements and the UTC time code. The first challenge was to detect aircraft arrivals from the anemometer data. The detection algorithm looks for the wake vortices that are likely to migrate towards the other parallel runway; the anemometers were placed only between the parallel runways. If the crosswind is directed from runway $25 \mathrm{~L}$ toward $25 \mathrm{R}$, then vortices will only be detected from aircraft landing on $25 \mathrm{~L}$, and vice versa. The ambient crosswind is estimated as the median of the 1-minute averaged crosswinds measured by the 10 anemometers. Since the wake vortices take some time to descend (aircraft at about 65 meters height) to the anemometer array (height of 15 meters), the largest vortex-induced crosswind is likely to occur at the second or third anemometer from the end of the array. The algorithm looks for the crosswind at the second or third anemometer to be at least $4 \mathrm{~m} / \mathrm{s}$ stronger than the ambient crosswind. (Various vortexinduced crosswind magnitudes were examined, but 4 $\mathrm{m} / \mathrm{s}$ was the smallest value that consistently identified vortices.)

To ensure that the downwind vortex translated to the second or third anemometer, a minimum ambient crosswind was set at $1 \mathrm{~m} / \mathrm{s}$ (or $-1 \mathrm{~m} / \mathrm{s}$, depending on the runway). Crosswinds less than $\pm 1 \mathrm{~m} / \mathrm{s}$ often did not lead to a vortex detection whereas crosswinds of at least $\pm 1 \mathrm{~m} / \mathrm{s}$ always yielded a detection.

Thus, two crosswind-based thresholds were employed. The crosswind magnitude had to be at least $1 \mathrm{~m} / \mathrm{s}$ to allow a vortex to reach the second anemometer and the vortex-induced crosswind had to be at least $4 \mathrm{~m} / \mathrm{s}$ stronger than the ambient crosswind to signal that a vortex was detected.

For each detected arrival, a run file was generated to save the data from 30 seconds before the detection until the next detection or 180 seconds after the detection, whichever came first. The aircraft arrival time was estimated to occur 10 seconds into the run file and subsequent analyses showed this to be surprisingly reasonable.

Figure 1 shows time history plots of the winds for a MD-11 landing. The top plot shows the 2-second average headwinds measured by the 10 ultrasonic anemometers. The middle plot shows the crosswinds at each anemometer; motion of the vortices across the array is apparent. The bottom plot shows the maximum, median, and minimum crosswinds across the array. 


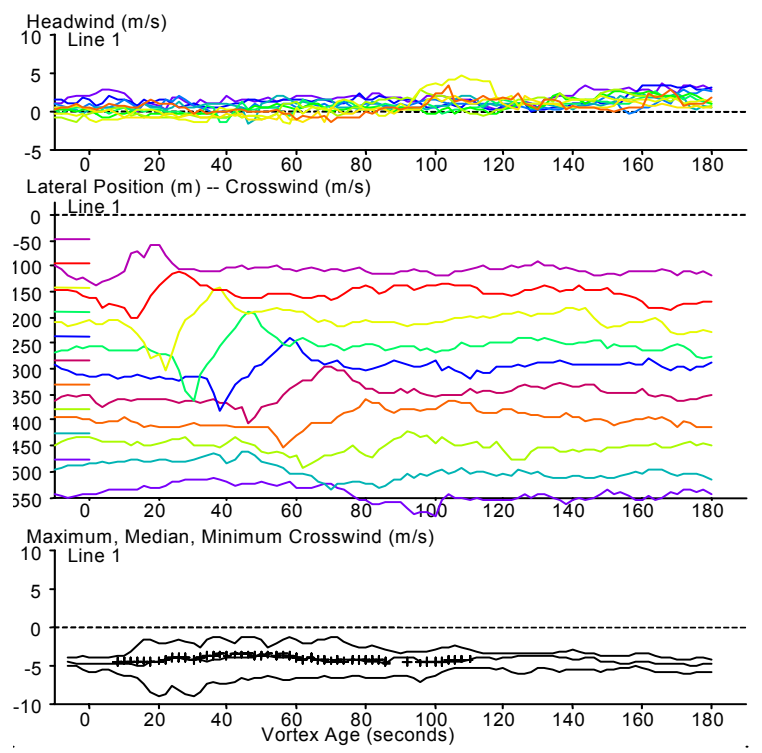

Figure 1. MD-11 Landing on 25R at Frankfurt/Main

From the vortex tracking point of view, the bottom plot is the most important. The automated routine estimates the vortex locations and demise based on this data. The maximum and minimum crosswinds are associated with the two wake vortices. The vortex-induced crosswind is taken as the difference between the maximum (or minimum) and the median. A least-square fit to the measured crosswind is plotted as "+" in the bottom plot.

When the ambient winds were low or at least exhibited little variation, the automated routine worked well. As the winds increased along with the attendant normal spatial and temporal variations, the routine was less reliable. Each run was visually checked and corrections made to the automated results when warranted. Here the crosswind values in the middle plot were used to visualize and determine or confirm the vortex location and demise time. A vortex was assumed to have expired when the vortex signal was comparable to the ambient wind. When the ambient winds were turbulent (gusts and/or rapid amplitude changes), the vortex was assumed to have expired when the vortex signal became less than any ambient wind signal within about 20-30 seconds of the expected (projected) vortex location.

The reduced data were entered into an Access database. The following data elements were captured for each run:

- Runway (25L or $25 \mathrm{R})$

- Aircraft Type (B-737, B-777, A-300, etc.)

- Date
- Time (UTC time, converted to local time in hours, minutes, and seconds)

- Port vortex age (demise time or time vortex exited the anemometer array)

- Port vortex distance (distance from extended runway centerline where vortex last detected)

- Starboard vortex age

- Starboard vortex distance

- Headwind (1-minute average at the time aircraft passed over the anemometer array)

- Crosswind (1-minute average at the time aircraft passed over the anemometer array)

- Turbulence indication (defined in section 4.3.4)

Other data were also recorded for each landing, but these were not used in the analyses reported below.

\section{VORTEX DATA ANALYSES}

In the analyses below, reference is often made to Vortex 1 (denoted V1) and Vortex 2 (V2). Vortex 1 is the downwind vortex (the first vortex detected by the anemometer array) and Vortex 2 is the upwind vortex, with respect to the crosswind. As one views the results, recall that the crosswind magnitude had to be at least $1 \mathrm{~m} / \mathrm{s}$, so a number of landings were ignored. Also, under low crosswind conditions (1-2 m/s) Vortex 2 oftentimes was not detected by the anemometer array (crosswind not strong enough to move the vortex close enough to be detected). In addition, under these crosswind conditions Vortex 2 sometimes was detected by the first anemometer only; when the signal decreased to the ambient wind the vortex either decayed near the anemometer or moved away from the array. Similarly, if a vortex was tracked moving across the entire array, the demise time was entered as the time when the vortex was no longer seen on the last anemometer. It either expired then or lasted longer. All these limitations are reflected in the analyses to be described below.

Table 1 shows the number of vortices (both V1 and V2) detected in April 1998 by aircraft type. The table also delineates the data by the runway on which the aircraft landed.

Table 1. Number of Vortices Detected

\begin{tabular}{|c|c|c|c|c|}
\hline $\begin{array}{c}\text { AIRCRAFT } \\
\text { TYPE }\end{array}$ & \multicolumn{2}{|c|}{ Runway 25L } & \multicolumn{2}{c|}{ Runway 25R } \\
\hline & V1 & V2 & V1 & V2 \\
\hline A300 & 140 & 127 & 42 & 28 \\
\hline A310 & 120 & 106 & 28 & 21 \\
\hline A319 & 101 & 89 & 15 & 15 \\
\hline
\end{tabular}




\begin{tabular}{|c|c|c|c|c|}
\hline A320 & 281 & 243 & 95 & 74 \\
\hline A321 & 203 & 172 & 35 & 27 \\
\hline A330 & 9 & 8 & 1 & 1 \\
\hline A340 & 47 & 47 & 15 & 10 \\
\hline B727 & 21 & 17 & 6 & 3 \\
\hline B737 & 358 & 310 & 89 & 70 \\
\hline B747 & 256 & 215 & 69 & 39 \\
\hline B757 & 54 & 50 & 17 & 13 \\
\hline B767 & 122 & 106 & 24 & 17 \\
\hline B777 & 14 & 10 & 5 & 1 \\
\hline BA46 & 21 & 16 & 3 & 1 \\
\hline DC10 & 37 & 31 & 5 & 4 \\
\hline DC9 & 6 & 6 & 0 & 0 \\
\hline IL86 & 11 & 8 & 2 & 2 \\
\hline L101 & 9 & 7 & 2 & 1 \\
\hline MD11 & 44 & 36 & 13 & 11 \\
\hline MD80 & 56 & 46 & 16 & 13 \\
\hline MD87 & 7 & 6 & 0 & 0 \\
\hline MD90 & 5 & 4 & 1 & 0 \\
\hline TU54 & 5 & 5 & 3 & 3 \\
\hline Totals & 1927 & 1665 & 486 & 354 \\
\hline
\end{tabular}

\subsection{Distance Translated}

The automated routines estimated the vortex demise time (vortex age) and the location where the vortex was last detected. Each landing was reviewed and either the automated values accepted or revised based on review of the vortex tracks (Figure 1). In the former case, the locations are the least-squares fit to the data; in the latter, the locations are the distance to the anemometer where the vortex was last detected.

Figures 2 to 5 show the distances the vortices translated as a function of the 1-minute average crosswind for B-737 and B-747 aircraft. The solid line on each graph is a least-squares fit to the data. As one might expect, V1 travels farther than V2 for a given crosswind. Figures 6 and 7 show the vortex survival probabilities for V1 and V2 and by landing runway. All aircraft are included in the plots. These figures clearly show that V1 translates farther than V2. One also sees that vortices from aircraft landing on runway $25 \mathrm{~L}$ travel farther than for runway $25 \mathrm{R}$ as the anemometer array is not perfectly perpendicular to the runways. For the same headwind magnitude, but crosswinds of different signs, vortices from $25 \mathrm{~L}$ are on average older than vortices from $25 \mathrm{R}$.

Looking at Figures 2 to 5, at a given distance D there is a distribution of average crosswinds translating the vortex to that maximum distance. Figure 6 shows the probability of a vortex translating a distance $\mathrm{D}$ on
Figure 2. Average Crosswind vs. V1 Distance, B737 Aircraft

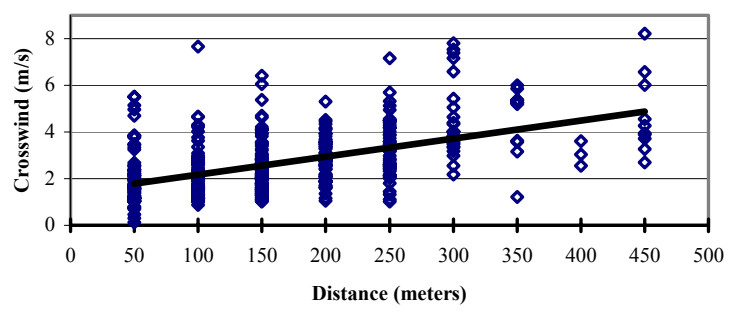

Figure 3. Average Crosswind vs. V2 Distance, B737 Aircraft

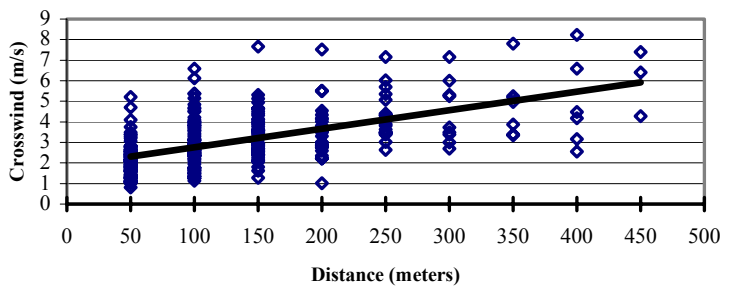

Figure 4. Average Crosswind vs. V1 Distance, B747 Aircraft

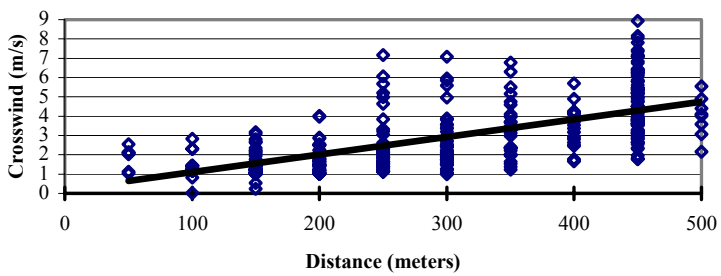

Figure 5. Average Crosswind vs. V2 Distance, B747 Aircraft

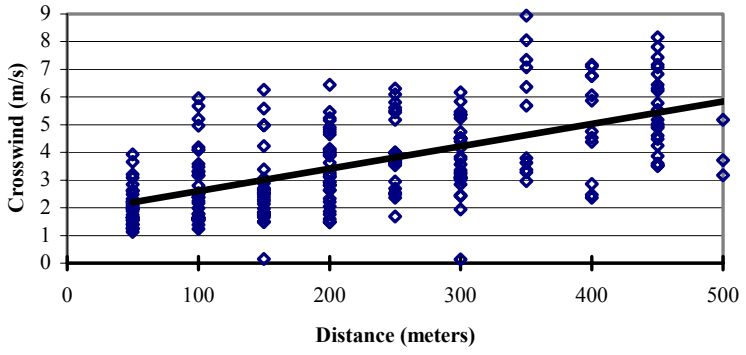

Runway 25L; Figures 8 and 9 show this probability as a function of $\mathrm{D}$ and of the square of $\mathrm{D}$, or an $\exp (-\gamma \mathrm{D})$ and an $\exp \left(-\alpha \mathrm{D}^{2}\right)$ dependence ${ }^{17}$, respectively. Note the $\mathrm{R}^{2}$ goodness-of-fit values, especially for the longer distances. 
Figure 6. V1 \& V2 Survival Probabilities At

Various Distances On 25L

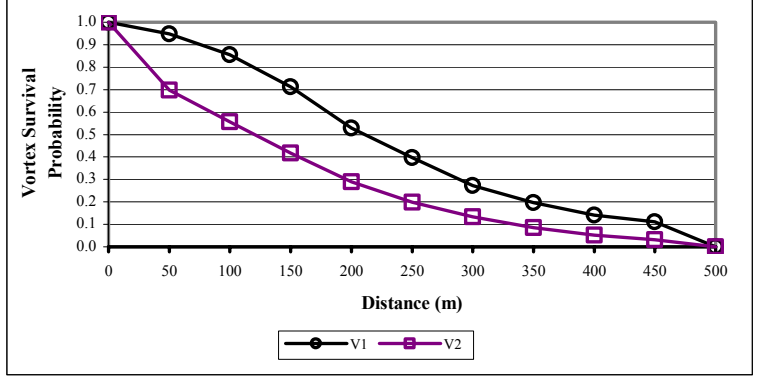

Figure 7. V1 \& V2 Survival Probabilities At Various Distances On 25R

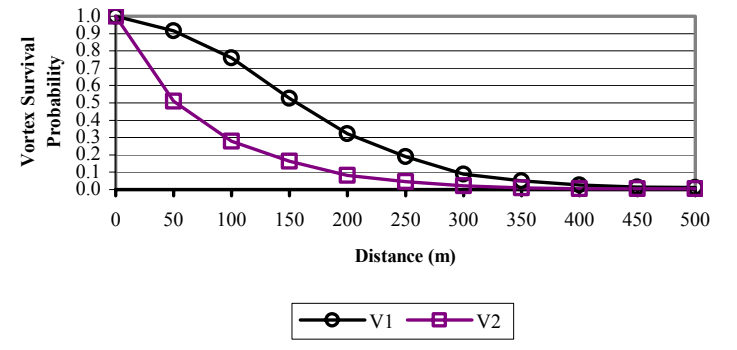

Figure 8. Frankfurt - Runway 25L, All Aircraft

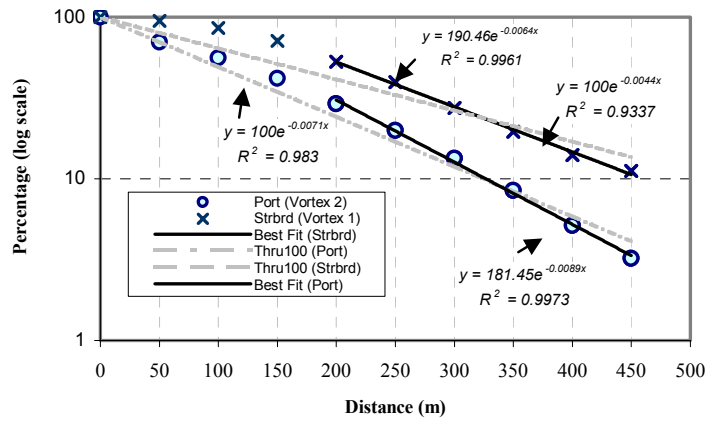

Figure 9. Frankfurt - Runway 25L, All Aircraft

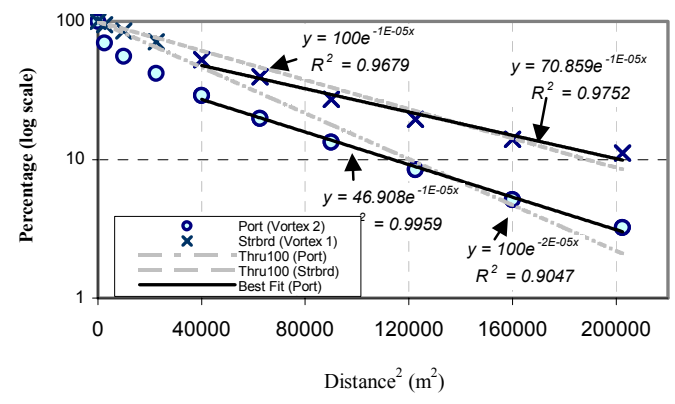

\section{$\underline{4.2 \text { Vortex Demise Time }}$}

Figure 10 shows the percentage of vortices (runway 25L, all aircraft) that survived as a function of vortex age. Now one sees that V2 lives longer than V1. In Figure 11 the percentage is shown plotted as a function of the square of the vortex age. Note the linear fit to the data, which shows a clear $\exp \left(-\beta \mathrm{t}^{2}\right)$ dependence. $^{17}$
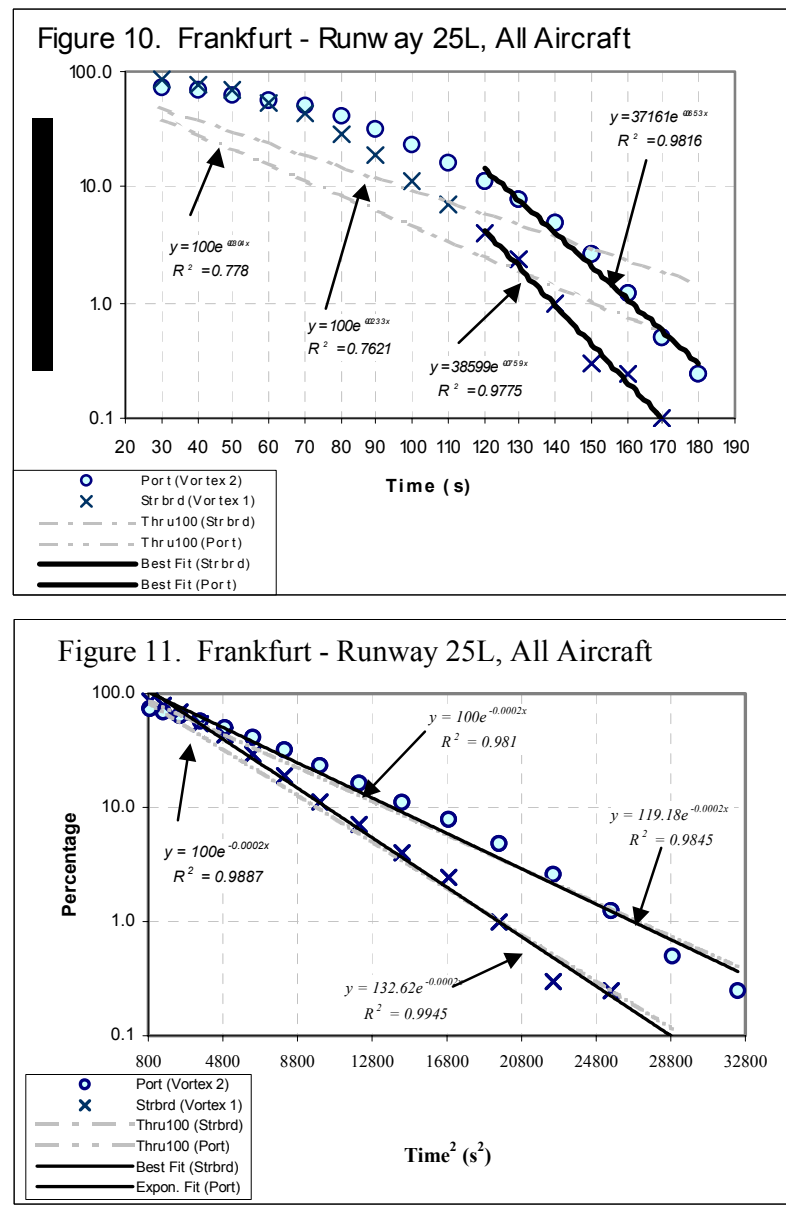

Figure 12. V1 Lifetime of B737 Aircraft in NonDimensional Time Units on 25L

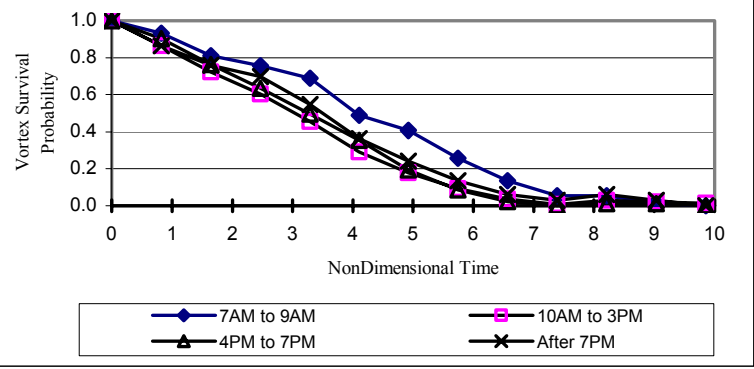

Figures 12 and 13 show the vortex survival probability plotted versus non-dimensional time (time for a vortex to descend a distance equal to the initial vortex spacing). The B-737 and B-747 used 12.2 and 23.6 
seconds, respectively, as the non-dimensional lifetimes.

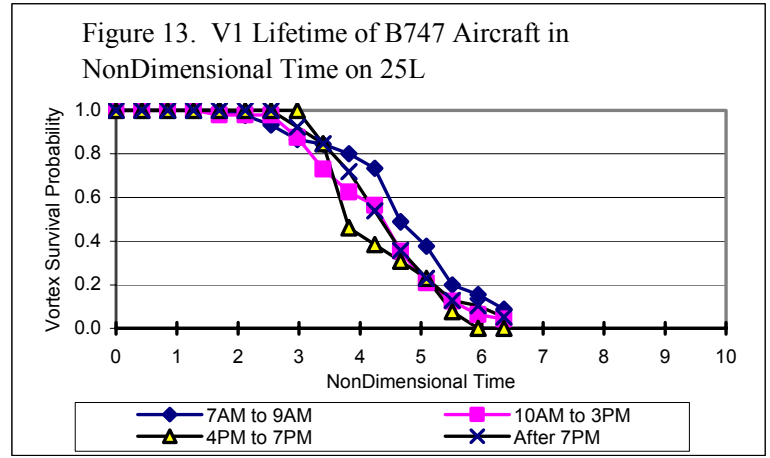

\subsection{Observations}

The general behavior of the wake vortices agrees with the results obtained in previous measurements. However, the sensitivity and height of the ultrasonic anemometers and the long tracking distance (500 meters) have yielded new insights into wake vortex behavior. The anemometer sensitivity and height permitted tracking the vortices even when the downwind vortex (V1) experienced the expected bounce $^{18}$ and increased its height above ground along with the attendant decay.

\section{$\underline{\text { 4.3.1 Time-of-Day Effect }}$}

It has long been suspected that the time of day should have an effect on the lifetime of a vortex. The expectation is that vortices will live longer in the early morning or late evening when the ambient turbulence is low compared to late morning and the afternoon when local heating should lead to higher turbulence levels. Figures 12 and 13 seem to indicate a time-ofday effect, especially for the early morning. Part of this effect might be attributed to varying load factors of the aircraft and requires further examination.

\subsubsection{Vortex Lifetimes}

Heavy aircraft vortices appear to have a Gaussian distribution of lifetimes centered at about 100 seconds. The crosswind component then indicates where the center of a Gaussian distribution of vortex demise distances is located. For Large aircraft vortices, there is a crosswind value beyond which there is no additional vortex transport; thus indicating that the lifetimes of Large aircraft vortices are dependent on the crosswind magnitude.

The translation of both $\mathrm{V} 1$ and $\mathrm{V} 2$ exhibited a relatively constant speed for about $3 / 4$ of their lifetime followed by a gradual slowing down to their end of life. Using the vortex demise distance divided by the lifetime as the vortex-induced speed, Figures 14 and 15 indicate that $\mathrm{V} 1$ was about $1 \mathrm{~m} / \mathrm{s}$ faster and $\mathrm{V} 2$ was about $1 \mathrm{~m} / \mathrm{s}$ slower than the crosswind. Many of the cases showing the opposite result were for short-lived vortices.

Figure 14. Average Crosswind vs. V1 on 25L

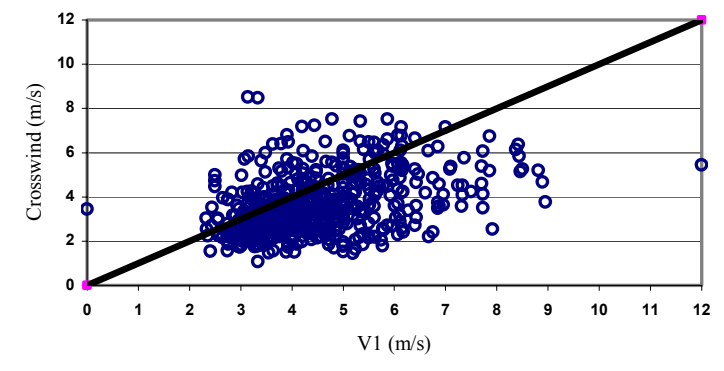

Figure 15. Average Crosswind vs. V2 on $25 \mathrm{~L}$

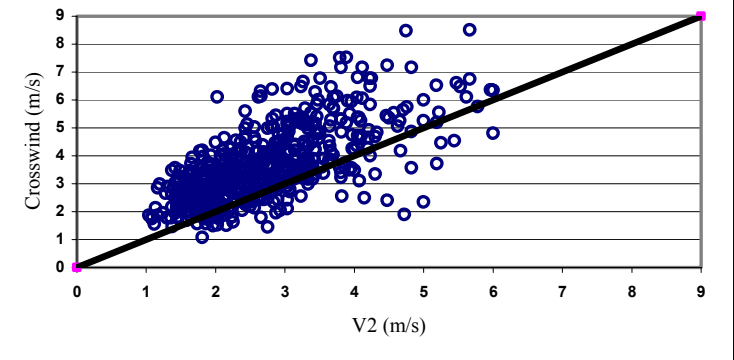

Figure 16. V1 \& V2 Lifetimes of A319

Aircraft In NonDimensional Time Units

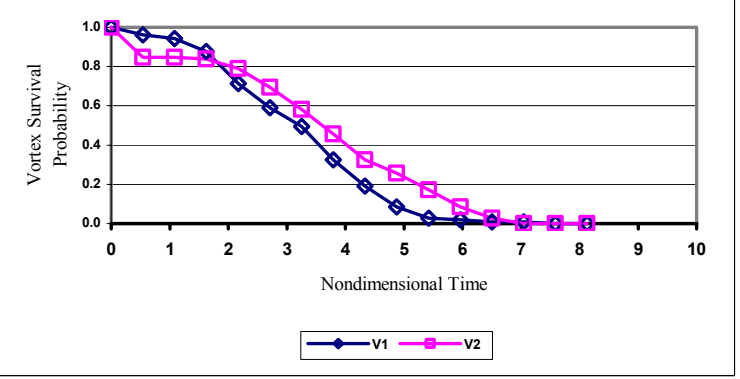

Figure 17. V1 \& V2 Lifetimes of A300

Aircraft In NonDimensional Time Units

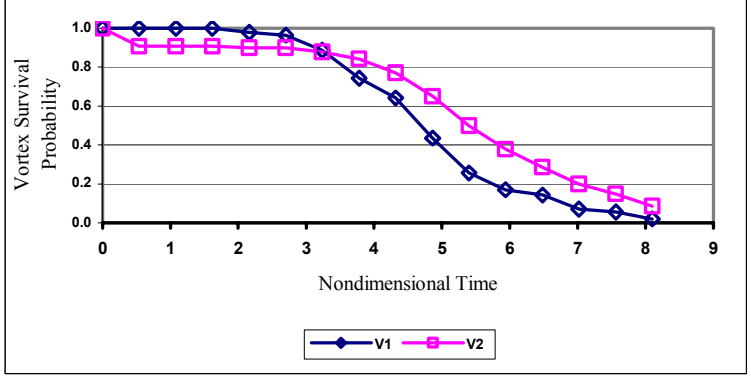


Figures 16 and 17 show the vortex lifetimes for the A-319 and A-300 in non-dimensional units (one unit is approximately 18 seconds for both aircraft types). The dip at about $1 / 2$ unit of non-dimensional time for V2 is caused by the minimum $1 \mathrm{~m} / \mathrm{s}$ crosswind restriction; about $10 \%$ of the landings did not record a V2 vortex or the V2 vortex was only detected on the sensor closest to the runway extended centerline and was excluded as the loss of the vortex signal could be either vortex demise or the vortex moving back toward the runway centerline and out of the range of the sensor. As also noted in Section 4.2 (Figures 10 and 11), V2 usually lives longer than V1. One might question this result based on the anemometer sensitivity in light of the familiar vortex bounce $^{18}$ (and the attendant vortex decay, which accompanies the bounce). The data analysis software does attempt a least-square fit to the vortex height. These results are not shown herein as they are very approximate, but the indicated heights are within limits where the vortices should be detected.

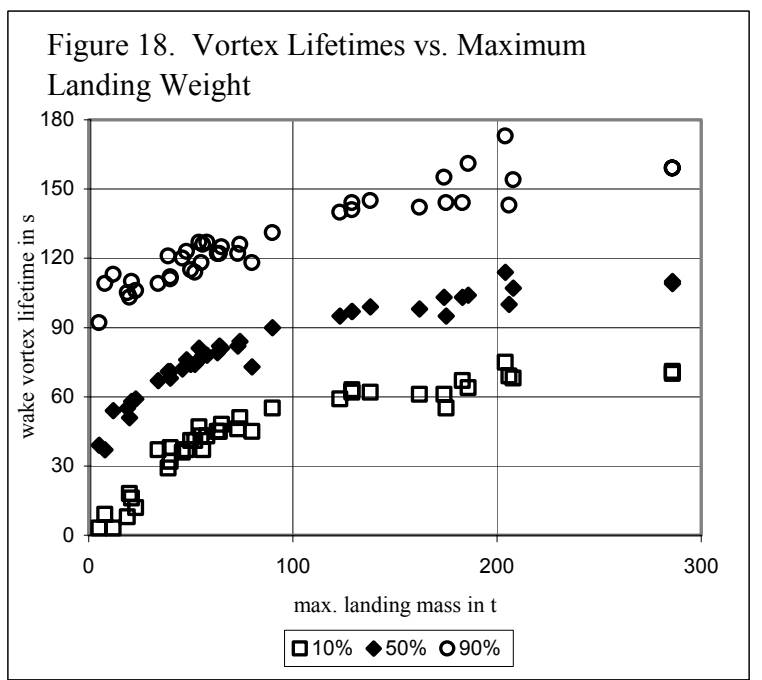

Figure 18 shows the $10 \%, 50 \%$, and $90 \%$ percentiles of the lifetime distribution for various aircraft ranging from a Learjet 35 up to the B-747-400. The analysis is based on a couple of assumptions that require further examination. These assumptions, however, are not expected to affect the results for different aircraft in a significantly different manner. There could be a constant offset, but as long as differences in vortex lifetimes of different aircraft types are considered these effects should cancel out. Therefore, the given absolute numbers shall be considered preliminary. Even more so, the fact that a vortex has been detected by the windline with low detection thresholds does not necessarily mean that there would be a hazard for following aircraft. The assumptions and limitations of the analysis under consideration are:
1. Only those aircraft types are displayed where at least 100 landings lead to proper detection and recognition of one or more vortices.

2. The instant when the aircraft passes the windline is not known exactly. Variations about \pm 20 $\mathrm{s}$ seem realistic.

3. The analysis refers to the maximum landing weight rather than the actual weight.

4. A landing has been analyzed only if the landing was on $25 \mathrm{~L}$ and the crosswind was positive (blowing from 25L to 25R) or vice versa.

5. There are events where a landing of an aircraft is indicated but there are no vortex signals recognized at the anemometers although the crosswind would transport the vortex towards the windline. This happens more frequently with light aircraft, for heavy aircraft the detection efficiency exceeds $95 \%$. These events have been excluded from the current analysis.

6. A small fraction of vortices are moving across the entire windline. Individual vortex lifetimes may be underestimated if the slower V2 will be transported by more than $500 \mathrm{~m}$. This small effect is certainly not causing the saturation of vortex lifetimes for the heavy aircraft.

7. The windline is not perpendicular to the runways which results in slightly different lifetime distributions for landings on $25 \mathrm{~L}$ and 25R. The present data has not yet been corrected for this effect.

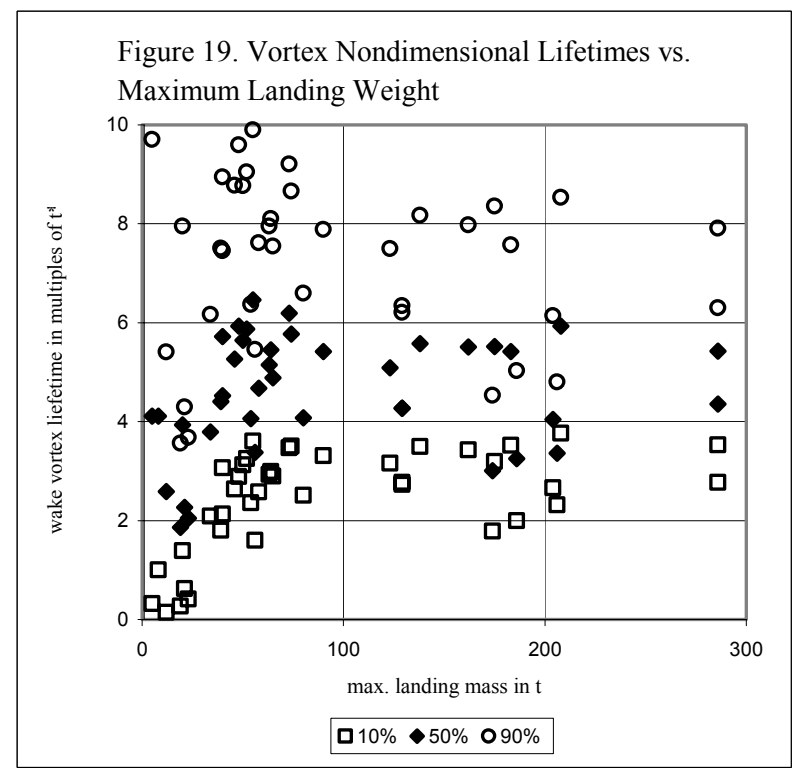

Nonetheless, several conclusions can be drawn from Figure 18 at this stage:

$>$ The clearly visible trend vanishes if the results are displayed in multiples of the nondimensional time 
$t^{*}$ (see Figure 19). In particular, a universal scaling of the decay with nondimensional time $t^{*}$ is not supported by this analysis of the in-groundeffect situation. This is corroborated by an additional observation:

$>$ There are pairs of aircraft having comparable mass but different wingspan and thus different nondimensional times $\mathrm{t}^{*}$. Small aircraft: Dash 8 and ATR-72 (large $t^{*}$ ) vs. Canadair Regionaljet (small $\left.\mathrm{t}^{*}\right)$; Large aircraft: Yakovlev 42 (large $\mathrm{t}^{*}$ ) vs. B-737 (small t*); Heavy aircraft: A-330 (large $\left.\mathrm{t}^{*}\right)$ vs. L-1011 and Ilyushin 86 (small $\mathrm{t}^{*}$ ). Nevertheless, significant differences in physical lifetimes of vortices are not observed in any of these pairs.

$>$ The B-757 vortices do not appear as extreme outliers, but behave like vortices from other aircraft of comparable size.

$>$ The saturation of vortex lifetimes for heavier aircraft indicates that on average vortices from aircraft larger than the B-747-400 should not be much longer lived than those of present heavy aircraft.

\subsubsection{Effect of Turbulence}

Atmospheric turbulence drives the sinuous instability mechanism and acts to erode vortices by redistributing the vorticity within the vortex. Usually, vortex lifetime is correlated with $\varepsilon$, the equilibrium rate of turbulent eddy dissipation. This works well for vortices at altitude and has been used with some success for vortices near the ground. ${ }^{7}, 19$ However, because of the presence of the ground, the inertial subrange concept might be inadequate.

Near the ground the effect of turbulence has not been clearly shown, although it is known that vortices decay more rapidly near the ground. ${ }^{19}$ Recall that Figure 17 shows that half of the vortices have decayed after about 5 times the non-dimensional lifetime. Figures 9 and 11 show that the vortex survival probability has both an $\exp \left(-\alpha \mathrm{D}^{2}\right)$ distance dependence and an $\exp \left(-\beta \mathrm{t}^{2}\right)$ time dependence. Thus, it appears that vortex decay near the ground is only weakly dependent on the meteorological conditions.

As stated in Section 3, a vortex is assumed to have expired when the vortex signal is comparable to the ambient crosswind. By this statement, one would, of course, expect a vortex to "expire" sooner when the ambient winds are fluctuating. The hazard posed by a vortex embedded in a fluctuating ambient wind is decreased significantly when the vortex plus fluctuating wind are integrated over the wingspan of an encountering aircraft. Thus, the effect of such a vortex cannot be distinguished from the effect of the turbulent or gusty wind.

To understand the role of turbulence near the ground, a less formal definition of turbulence is used. Refer to the top plot of Figure 1 which shows the headwind measured at the 10 anemometer locations. The ambient wind is defined herein as "turbulent" when the headwind plots show a band of at least $3 \mathrm{~m} / \mathrm{s}$ width (the case in Figure 1 was not deemed turbulent, although after 160 seconds it did reach $3 \mathrm{~m} / \mathrm{s}$ ). Although not easily seen in the middle plot of Figure 1 , the original plots were large enough that a similar 3 $\mathrm{m} / \mathrm{s}$ difference in crosswind could also be detected. When the headwinds and/or crosswinds exhibited a 3 $\mathrm{m} / \mathrm{s}$ difference across the anemometer network, the data file (the landing) was flagged as a turbulent case.

Figures 20 and 21 show the vortex survival probability for $\mathrm{V} 1$ and $\mathrm{V} 2$, in turbulent and nonturbulent conditions, for the B-737 and B-747, respectively. In general, the turbulent cases exhibited about a 1 nondimensional time unit more rapid decay than the non-turbulent. The V1 turbulent cases showed the most rapid decay. Although not shown here, the A-300, A-310, A-319, and A-320 showed the same results. (A review of previous data collection efforts ${ }^{3,7,19}$ showed a similar result.)

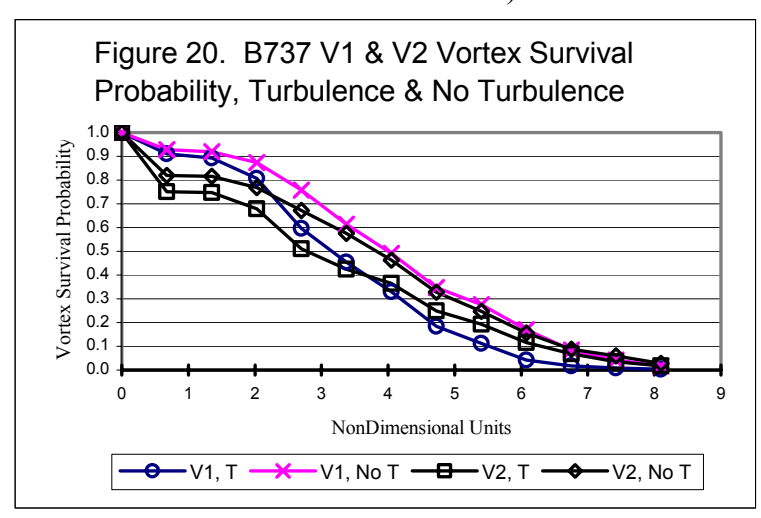

Figure 21. B747 V1 \& V2 Vortex Survival Probability, Turbulence \& No Turbulence

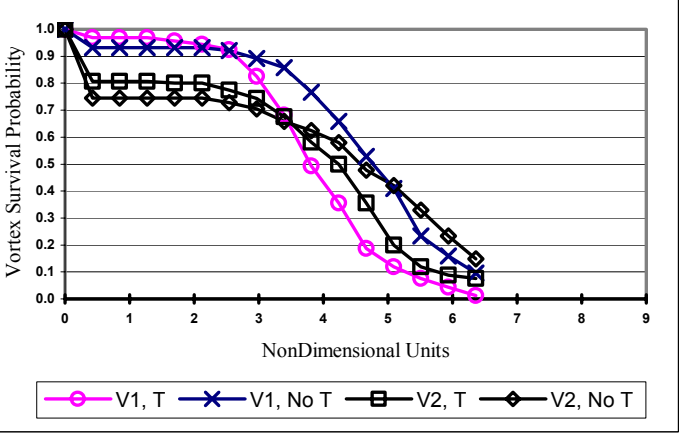


But, does this mean that vortices in turbulent conditions expire sooner than vortices in no turbulence conditions? Figure 1 shows a case with little turbulence; the vortex-induced signature, in particular the maximum and minimum crosswinds, is clear. Note that nothing is being said about the hazard of these vortices. The anemometers are very sensitive and it is expected that the vortex circulations are too weak at the farthest distance to affect an aircraft encountering these vortices.

Although difficult to see in these small figures, a remnant vortex is usually seen in the turbulent cases. That is, after the vortex demise (vortex signal less than ambient winds) the vortex still can be seen within the background winds. Although difficult to measure accurately, it appears that the vortex remnant lasts about 1 non-dimensional time unit. Thus, it appears that vortices decay stochastically with ambient turbulence masking the vortex (and, hence, its potential effect on an encountering aircraft) producing an "effective lifetime" shortened by about 1 nondimensional time unit.

\section{$\underline{\text { 4.3.4 Other Observations }}$}

There were events that require further study, but are noted here. There were rare occasions when a vortex signal was missing on 1 or 2 adjacent sensors yet continued on subsequent sensors. For example, the vortex was clearly seen at poles $1,2,3,4,6,7$, and 8 . Pole 5 was in operation (previous and subsequent landings yielded good signals at that location).

Recall that the anemometer array was not perpendicular to the extended runway centerline. [After checking data collected at other airports using anemometer arrays that were perpendicular to the centerline, similar events were noted but with less frequency.] Perhaps these events occur when the vortex links with the ground (the Crow instability) at the sensor distance.

Another observation currently being studied concerns temperature measurements near the vortex. The anemometers on each pole also measure temperature. Oftentimes (the frequency is under study) the temperature decreased by $1^{\circ} \mathrm{C}$ as a vortex passed over the pole. The passage of wind gusts did not indicate any temperature change. It is postulated that cooler air at the aircraft altitude (65 meters) is captured within the wake oval and it is the cooler air surrounding the vortex that is measured.

\section{IMPACT ON PARALLEL RUNWAY OPERATIONS}

The Frankfurt data collection system monitored wake vortex motion and decay over an extent of approximately 500 meters roughly perpendicular to the extended runway centerlines at a location approximately 900 meters from the runway threshold. Landing aircraft passed over the anemometer array at a nominal altitude of 65 meters. Based on the approximately 2500 landings in April 1998, five effects were established that affect how the capacity of parallel runways might be increased. A more automated means of data reduction and analysis is being developed to increase the data coverage (other seasons of the year) and to improve the statistics as 2500 landings is not sufficient to justify changing procedures.

First, V1 decays faster than V2. This has been suggested by other studies, but is explicitly shown herein. V1 should decay faster as it is the vortex which has been observed to bounce (increase in altitude); the mechanism for the bounce ${ }^{18}$ leads to a more rapid decay. Since V1 is the vortex that could affect operations on a downwind parallel runway, the faster decay is important as it should lead to both a maximum lifetime (and, hence, a minimum parallel runway separation) dependent on aircraft mix and crosswind magnitude. Note that the vortex hazard is not addressed in this study, but will lead to shorter lifetimes and lateral distances.

Second, the distance vortices move is dependent on the crosswind magnitude. For Large (Medium) aircraft this study indicates there is a crosswind value beyond which there is no additional vortex transport; such a value should exist for Heavy aircraft, but was not observed as a small percentage of vortices from Heavy aircraft translated more than 500 meters. Setting restrictions on crosswind magnitude will lead to vortex-free operations for closely spaced parallel runways.

Third, ambient turbulence was shown to play a lesser role in the demise of vortices near the ground compared to vortices out of ground effect. Vortices do decay faster near the ground, but the mechanisms are due to the presence of the ground itself (boundary layer effects).

Fourth, temperature measurements near a suspected vortex might lead to a dependable means to distinguish a vortex from a wind gust. This could be important if an anemometer array is included as part of 
a wake vortex spacing or warning system, both for single runway and parallel runway operations.

Fifth, the vortex decay as a function of the maximum landing weight as shown in Figure 18 indicates that one can make a reasonable estimate of the vortex behavior for aircraft not observed in the Frankfurt data collection. For example, an initial estimate of the vortex behavior of the A-380 and the extended range B-747-400 can be extrapolated. The actual behavior will need to be measured, but the estimates can be used to determine the likely impact of these very heavy aircraft in single and parallel runway operations.

\section{REFERENCES}

1 Hallock, J. N., "Monitoring the Movement of Wake Vortices at Kennedy and Stapleton Airports," Proceedings of the Fifth Annual Symposium of the Society of Flight Test Engineers, Anaheim, CA, 1974, pp. 4/7-4/12.

2 Hallock, J. N., Wood, W. D. and Spitzer, E. A., "The Motion of Wake Vortices in the Terminal Environment," Proceedings of the Sixth Conference on Aerospace and Aeronautical Meteorology, American Meteorological Society, 1974, pp. 393-398.

3 Sullivan, T. E., Hallock, J. N. and Winston, B. P., "Analysis of Ground-Wind Vortex Sensing System Data from O'Hare International Airport," FAA-RD80-133, Sept. 1980, DOT Transportation Systems Center, Cambridge, MA.

4 Sullivan, T. E., Hallock, J. N., Winston, B. P., McWilliams, I. G. and Burnham, D. C., "Aircraft Wake Vortex Takeoff Tests at Toronto International Airport," FAA-RD-78-143, Feb. 1979, DOT Transportation Systems Center, Cambridge, MA.

5 Campbell, S. D., Dasey, T. J., Freehart, R. E., Heinrichs, R. M., Matthews, M. P. and Perras, G. H., "Wake Vortex Field Measurements Program at Memphis, TN," AIAA Paper No. 96-1399, Reno, NV, Jan. 1996.

6 Krause, M. C., Eberle, W. R., Miller, G. M. and Gorzynski, E. J., "Investigation of Wind Conditions During Early Morning Hours at Los Angeles International Airport," FAA-RD-77-116, Oct. 1977, Lockheed Missiles \& Space Co., Huntsville, AL.

7 Hallock, J. N., Winston, B. P., Burnham, D. C., Sullivan, T. E., McWilliams, I. G., and Wood, W. D., "Joint US/UK Vortex Tracking Program at Heathrow
International Airport, Vol. II: Data Analysis," FAARD-76-58, II, DOT Transportation Systems Center, Cambridge, MA.

8 van Woortman, H. J. B. and Polak, F. R., "A Vortex Advisory System at Schiphol/Amsterdam Airport: Feasible and Meaningful?," Proceedings of the Aircraft Wake Vortices Conference, DOT/FAA/SD-92/1.1, DOT Volpe National Transportation Systems Center, Cambridge, MA, June 1992, pp. 5-1 - 5-15.

9 Rutishauser, D. K., and O'Connor, C. J., “Aircraft Wake Vortex Spacing System (AVOSS) Performance Update and Validation Study," NASA TM-2001211240, Oct. 2001, NASA Langley Research Center, Hampton, VA.

10 Harris, M., Vaughan, J. M., Huenecke, K. and Huenecke, C., "Aircraft Wake Vortices: a Comparison of Wind-Tunnel Data with Field Trial Measurements by Laser Radar," Aerospace Science and Tech., Vol. 4, 2000, pp. 363-370.

11 Tetzlaff, G., Franke, J. and Schilling, V., "Wake Vortex Propagation in the Atmospheric Boundary Layer," Proceedings of the Aircraft Wake Vortices Conf., DOT/FAA/SD-92/1.2, DOT Volpe National Transportation Systems Center, Cambridge, MA, June 1992, pp. 47-1 - 47-19.

12 Spitzer, E. A., Rudis, R. P., Hallock, J. N. and Greene, G. C., "Windline for Parallel Runway Operations at SFO," XXV General Assembly, European Geophysical Society, Nice, France, April 2000 .

13 Gurke, T. and Lafferton, H., "The Development of the Wake Vortices Warning System for Frankfurt Airport: Theory and Implementation," Air Traffic Control Quarterly, Vol. 5, No. 1, 1997, pp. 3-29.

14 Köpp, F., "Doppler Lidar Investigation of Wake Vortex Transport Between Closely Spaced Parallel Runways," AIAA Journal, Vol. 32, No. 4, April 1994, pp. 805-810.

15 Frech, M., Holzäpfel, F., Gerz, T. and Konopka, J., "Short-term Prediction of the Horizontal Wind Vector Within a Wake Vortex Warning System," Meteorological Applications, Vol. 9, 2002, pp. 9-20.

16 Abramson, S. and Burnham, D. C., "GroundBased Anemometer Measurements of Wake Vortices From Landing Aircraft," AGARD, CP-584, Trondheim, Norway, May 1996, pp. 13-1 to 13-7. 
17 Burnham, D. C. and Hallock, J. N., "Motion of Aircraft Wake Vortices in Ground Effect," DOT/FAA/AAR-00/16, April 2000, Volpe National Transportation Systems Center, Cambridge, MA.

18 Burnham, D. C., "Effect of Ground Wind Shear on Aircraft Trailing Vortices," AIAA Journal, Vol. 10, No. 8, August 1972, pp. 1114-1115.

19 Burnham, D. C. and Hallock, J. N., "Measurements of Wake Vortices Interacting with the Ground," AIAA paper No. 98-0593, Reno, NV, Jan. 1998. 